\title{
The Use of Non-natural Nucleotides to Probe Template- Independent DNA Synthesis
}

\author{
Anthony J. Berdis \\ Cleveland State University, A.BERDIS@csuohio.edu \\ David McCutcheon \\ Case Western Reserve University
}

Follow this and additional works at: https://engagedscholarship.csuohio.edu/scichem_facpub

Part of the Biochemistry Commons, and the Chemistry Commons

How does access to this work benefit you? Let us know!

\section{Publisher's Statement}

This is the accepted version of the following article: Berdis, A. J.; McCutcheon, D. The Use of Non-natural Nucleotides to Probe Template-Independent DNA Synthesis. ChemBioChem 2007, 8, 1399-1408., which has been published in final form at http://onlinelibrary.wiley.com/doi/ 10.1002/cbic.200700096/abstract

\section{Recommended Citation}

Berdis, Anthony J. and McCutcheon, David, "The Use of Non-natural Nucleotides to Probe TemplateIndependent DNA Synthesis" (2007). Chemistry Faculty Publications. 223.

https://engagedscholarship.csuohio.edu/scichem_facpub/223

This Article is brought to you for free and open access by the Chemistry Department at EngagedScholarship@CSU. It has been accepted for inclusion in Chemistry Faculty Publications by an authorized administrator of EngagedScholarship@CSU. For more information, please contact library.es@csuohio.edu. 


\title{
The Use of Non-natural Nucleotides to Probe Template-Independent DNA Synthesis
}

\author{
Anthony J. Berdis and David Mccutcheon
}

The vast majority of DNA polymerases use the complementary templating strand of DNA to guide each nucleotide incorporation. There are instances, however, in which polymerases can efficiently incorporate nucleotides in the absence of templating information. This process, known as translesion DNA synthesis, can alter the proper genetic code of an organism. To further elucidate the mechanism of template-independent DNA synthesis, we monitored the incorporation of various nucleotides at the "bluntend" of duplex DNA by the high-fidelity bacteriophage T4 DNA polymerase. Although natural nucleotides are not incorporated at the blunt-end, a limited subset of non-natural indolyl analogues containing extensive :n:-eledron surface areas are efficiently utilized by the T4 DNA polymerase. These analogues possess high binding affinities that are remarkably similar to those measured during incorporation opposite an abasic site. In contrast, the $k_{p o t}$ values are significantly lower during blunt-end extension

\section{Introduction}

The vast majority of DNA polymerases catalyze the polymerization of deoxyribonucleotides by using the complementary templating strand to guide each incorporation. In this respect, polymerases are enigmatic as they maintain a remarkable degree of specificity despite the fact that the substrate changes during each cycle of dNTP incorporation due to the heteropolymeric nature of DNA. High-fidelity polymerases, such as the bacteriophage T4 DNA polymerase, maintain an extraordinary degree of selectivity by inserting only one of four potential dNTPs opposite a templating nucleobase. However, they are flexible enough to recognize four distinct pairing partners. Most models accounting for this selectivity invoke the formation of Watson-Crick hydrogen-bonding interactions between the incoming nucleotide with the templating nucleobase ${ }^{1}$ However; other molecular forces, such as electrostatic interactions, steric constraints, and desolvation properties of the nucleotide, likely play important roles during the polymerization cycle.

Several laboratories have attempted to define the role of these forces by using various non-natural nucleotides. For example, the Bergstrom and Davisson laboratories used azoleand triazole-carboxamide nucleotides to evaluate electrostatic contributions on polymerization efficiency.12 The Romesberg and Schultz laboratories developed hydrophobic nucleotides to explore the role of desolvation energies during replication. ${ }^{3}$ Furthermore, the Kool laboratory has used 2,4-difluorotoluene and other isosteric analogues of thymine to probe the role of when compored to incorporation opposite an abasic site. These kinetic differences suggest that the single-stranded region of the DNA template plays an important role during polymerization through stacking interactions with downstream bases, interactions with key amino acid residues, or bath. In addition, we demonstrate that terminal deoxynucleotide transferase, a templateindependent enzyme, can efficiently incorporate many of these non-natural nucleotides. However, that this unique polymerase cannot extend large, bulky non-natural nucleotides suggests that elongation is limited by steric constraints imposed by structural features present within the polymerase. Regardless, the kinetic data obtained from using either DNA polymerase indicate that template-independent synthesis can occur without the contributions of hydrogen-banding interactions and suggest that n:electron interactions play an important role in polymerization efficiency when templating information is not present.

shape complementarity for nucleotide selection during polymerization.14 Finally, our efforts have focused on the contributions of the rt-electron surface area of the incoming nucleotide on the dynamics of the DNA polymerization cycle, specifically with regards to the misreplication of damaged DNA. ${ }^{5}$.

Toward this goal, we have studied the mechanism of translesion DNA synthesis using the abasic site ${ }^{15} 1$ and the thymine dimer $^{161}$ as prototypical DNA lesions (Scheme $1 \mathrm{~A}$ ). The non-natural nucleotides illustrated in Scheme 1B were used to develop a structure-activity relationship describing the biophysical forces influencing nucleotide incorporation opposite these noninstructional DNA lesions. In general, non-natural analogues containing large rt-electron surface areas-such as 5nitro-indolyl-2'-deoxyriboside triphosphate (5-NITP), 5-phenylindolyl-2'-deoxyriboside triphosphate (5-PhITP), and 5-napthylindolyl-2'-deoxyriboside triphosphate (5-NapITP)-are incorporated -1000 times more efficiently than analogues such as 5amino-indolyl-2'-deoxyriboside triphosphate (5-AITP), 5-fluoroindolyl-2'-deoxyriboside triphosphate (5-FITP), and 5-cyclohex- 
yl-indolyl-2' deoxyriboside triphosphate (5-CHITP), which possess smaller n-electron surface areas.[sc, ${ }^{d} \mathrm{~J}$ The differences in catalytic efficiencies were caused by alterations in binding affinity ( $K_{d}$ effect) of the nucleotides as well as maximal rate constant for incorporation $\left(k_{p o l}\right.$ effect) $\mathrm{P}^{1}$ The binding affinity of the incoming nucleotide was influenced by its ability to fill the "void" present at either DNA lesion. In contrast to binding affinity, the $k_{p o l}$ values are highly sensitive to the nature of the DNA lesion. In this respect, lower values are measured during incorporation opposite a thymine dimer than opposite an abasic site. These differences suggest that the dynamics of nucleotide incorporation are limited by repositioning of the templating nucleobase into a proper conformation. As such, the rate constants for nucleotide incorporation at an abasic site are significantly faster since the lack of a templating nucleobase circumvents the need for repositioning.

This report further evaluates this mechanism by quantifying the ability of the T4 DNA polymerase (designated gp43) to catalyze template-independent DNA synthesis at the "bluntend" of duplex DNA. As illustrated in Scheme 2, nucleotide incorporation at the blunt-end is similar to translesion DNA synthesis, since both reactions occur in the absence of direct coding information. However, blunt-end extension is different since the lack of the downstream templating strand precludes possible stacking interactions of the incoming nucleotide with downstream bases. This difference provides an indirect way to evaluate whether polymerization efficiency is influenced by stacking interactions with the downstream template strand versus the penulti-
A)

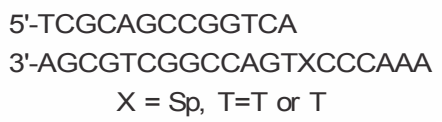

\section{$\mathrm{ob}$ \\ 2-hydroxymethyl-3- hydroxytetrahydrofuran}

$(\mathrm{Sp})$

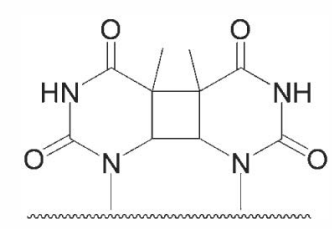

thymine dimer $(\mathrm{T}=\mathrm{T})$
B)

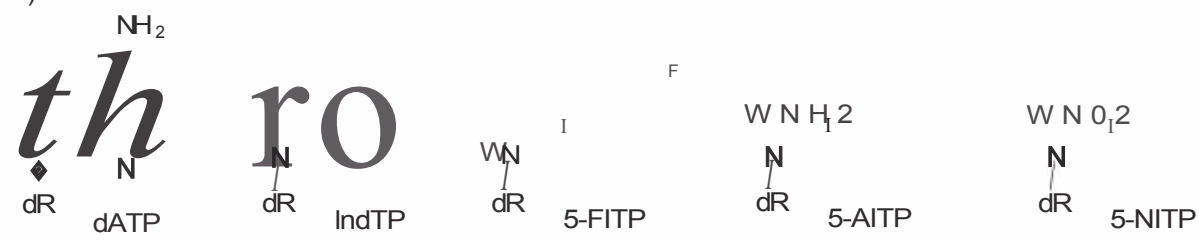

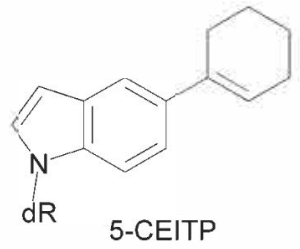

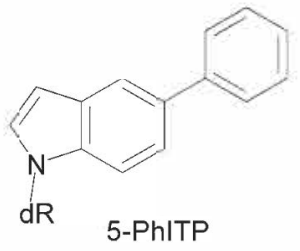

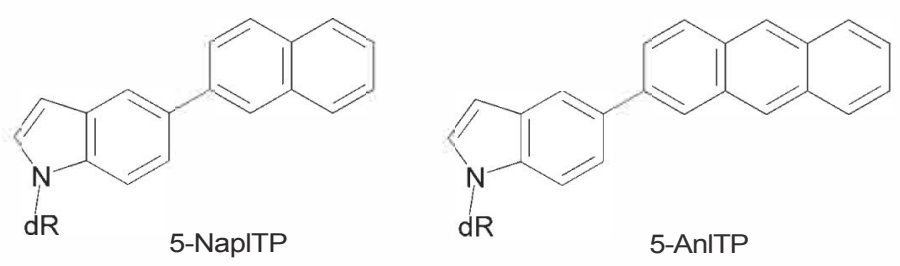

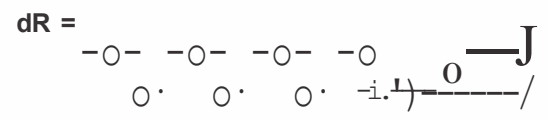

$\mathrm{OH}$

Scheme 1 A) Defined DNA substrates used for kinetic analysis. " $X$ " in the template strand denotes a tetrahydrofur an moiety designed to functionally mimic an abasic site, a thymine dimer, or thymine. B) Structures of 2 deoxynu cleoside triphosphates used or referred to in this study. For convenience, $\mathrm{dR}$ s used to represent the deoxyribose triphosphate portion of the nucleotides.

A)

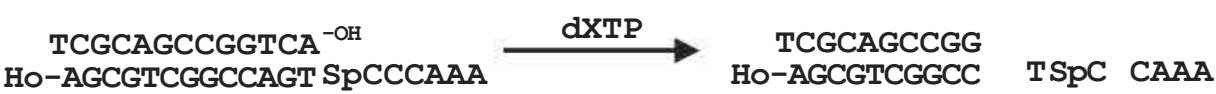

B)
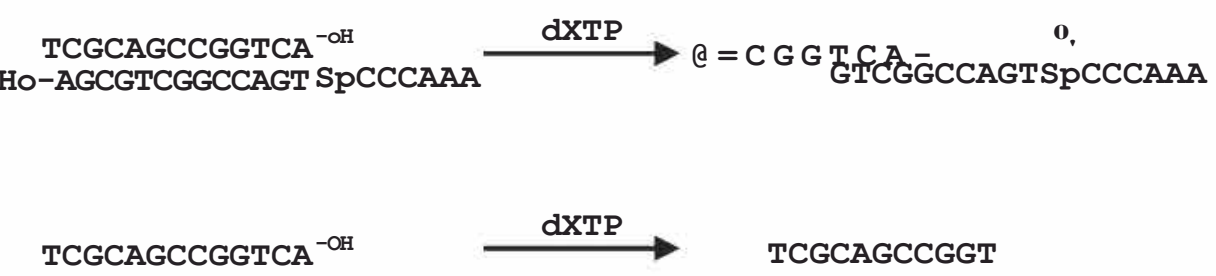

Scheme 2. Schematic representation of the three modes of template independent DNA synthesis. A) Nucleotide incorporation opposite an abasic site. B) Nucleotide incorporation at the blunt end of DNA Q Nucleotide incorpo ration by using single stranded DNA 
mate base pair. The studies reported here provide evidence that the overall efficiency of blunt-end extension is dependent upon the presence of n-electron surface area on the incoming nucleotide and recapitulates data reported for nucleotide incorporation opposite an abasic site.IsJ

\section{Results and Discussion}

\section{Nucleotide survey analyses}

The ability of the exonuclease-deficient bacteriophage T4 DNA polymerase, gp43 exo-, to perform template-independent DNA synthesis was evaluated by measuring the incorporation of the various non-natural indolyl nucleotides depicted in Scheme $1 \mathrm{~B}$ at the blunt-end of duplex DNA (Scheme 1A). Under these conditions, the extension of 20-mer to 21-mer was monitored as a function of time rather than extension of the 13-mer to 14-mer as typically measured during templatedependent DNA synthesis. Representative data provided in Figure 1 reveal that a limited subset of indolyl analogues is in-

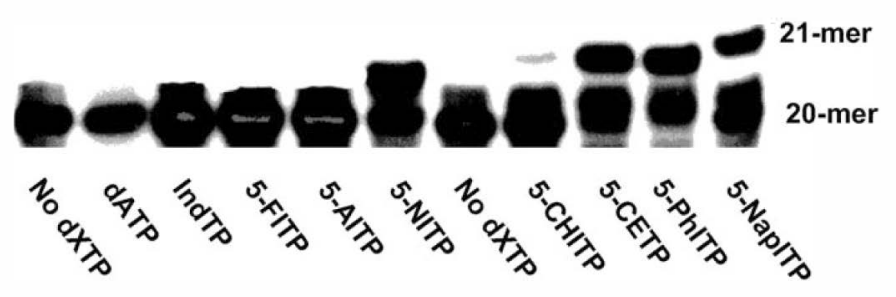

Figure 1. Gel electrophoresis data comparing the efficiency of blunt end ex tension by using natural and non natural nucleoside triphosphates. Assays were performed as described in the text.

corporated at the blunt-end of DNA. In general, analogues containing extensive n-electron surface areas are incorporated more efficiently than analogues that have smaller n-electron surface areas. It should be noted that natural nucleotides such as dATP and dGTP are not incorporated at the blunt-end of DNA, even at dNTP concentrations up to $2 \mathrm{mM}$ (data not shown).

\section{Kinetic parameters for blunt-end incorporation}

The kinetic dissociation constant, $\mathrm{K}_{\mathrm{d}}$, and maximal polymerization rate constant, $\mathrm{k}_{\mathrm{p} 01}$ for the incorporation of non-natural nucleotides during template-independent DNA synthesis were measured by using single-turnover reaction conditions, as previously described.[saJ Representative data provided in Figure 2A shows the dependency of the rate of enzymatic incorporation on 5-PhITP concentration. All time courses are best defined as a single-exponential process and were fitted to Equation (1) to define $\mathrm{k}_{a b}$, the rate constant of product formation. The plot of $\mathrm{k}_{a b}$, versus 5-PhITP concentration is hyperbolic (Figure $2 \mathrm{BJ}$, and a fit of the data to the Michaelis-Menten equation yields a $k_{\mathrm{p} 01}$ of $0.077 \pm 0.004 \mathrm{~s}-1, a \mathrm{~K}_{d}$ of $45 \pm 8 \mu \mathrm{M}$, and $a \mathrm{k}_{\mathrm{p} \alpha} / \mathrm{K}_{\mathrm{d}}$ value of $1710 \mathrm{M} 1 \mathrm{~s}-1 \cdot$ Similar analyses were performed for the other
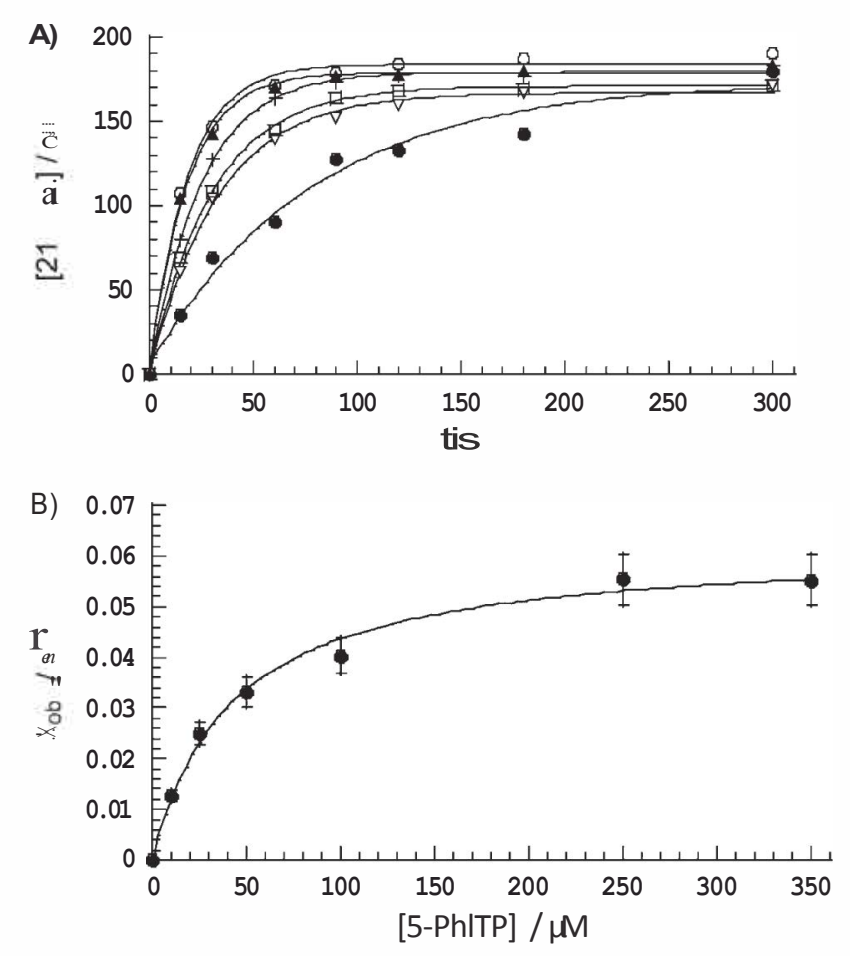

Figure 2. A) Dependency of the apparent rate constant for blunt end exten sion on the concentration of 5 PhITP as measured under single turnover conditions. Assays were performed with $1 \mu \mathrm{M}$ gp43 exo , $250 \mathrm{nM}$ 13/20 mer, $10 \mathrm{mM} \mathrm{Mg}(\mathrm{OAc})_{2}$, and $5 \mathrm{PhITP}$ in variable concentrations: $10 \mu \mathrm{M}(\mathrm{e}), 25 \mu \mathrm{M}$ (v), $50 \mu \mathrm{M}$ (o), $100 \mu \mathrm{M}(+), 250 \mu \mathrm{M}$ (\&), and $350 \mu \mathrm{M}$ (o). The solid lines rep resent the fit of the data to a single exponential. B) The observed rate con stants for incorporation (e) were plotted against 5 PhITP concentration and fitted to the Michaelis Menten equation to determine values corresponding to $\mathrm{Kd}$ and $\mathrm{k}_{\mathrm{po}}$.

non-natural nucleotides illustrated in Scheme $1 \mathrm{~B}$, and values corresponding to $\mathrm{K}_{\mathrm{d}}, \mathrm{K}_{\mathrm{p} 01}$ and $\mathrm{k}_{\mathrm{p} 0 \mathrm{i}} / \mathrm{K}_{\mathfrak{d}}$ are summarized in Table 1

Close inspection of the kinetic parameters for blunt-end extension versus incorporation at an abasic site reveal striking similarities and differences that provide useful insight into the mechanism of polymerization. In general, the $K_{d}$ values for analogues possessing significant $n$-electron density are nearly identical regardless of whether they are measured at the blunt-end or opposite an abasic site.IsJ Since these analogues differ significantly with respect to biophysical features such as size, shape, dipole moment, and solvation energies (see the Supporting Information), this suggests that the binding affinity for these nucleotides is influenced by their n-electron surface areas. One major difference, however, is that the $k_{p} d$ values for these analogues are significantly lower during blunt-end extension than for incorporation opposite an abasic site. For example, the $k_{p} d$ of $0.015 \mathrm{~s}-1$ measured for 5-NITP incorporation at the blunt-end of DNA is 8400 times slower than the $k_{p} d$ of $126 \mathrm{~s}-1$ measured opposite an abasic site.lsal Indeed, it is surprising that the value of $0.015 \mathrm{~s}-1$ measured for blunt-end extension is actually 60 times slower than the rate constant of 0.9 s-1 measured for insertion opposite a templating nucleobases such as thymine.IsaJ This kinetic trend is not limited to 5 NITP, as the $k_{P 01}$ values for other analogues such as 5-CEITP, 5PhITP, and 5-NapITP also show the preferential order of incorporation of abasic site templating base blunt end. 


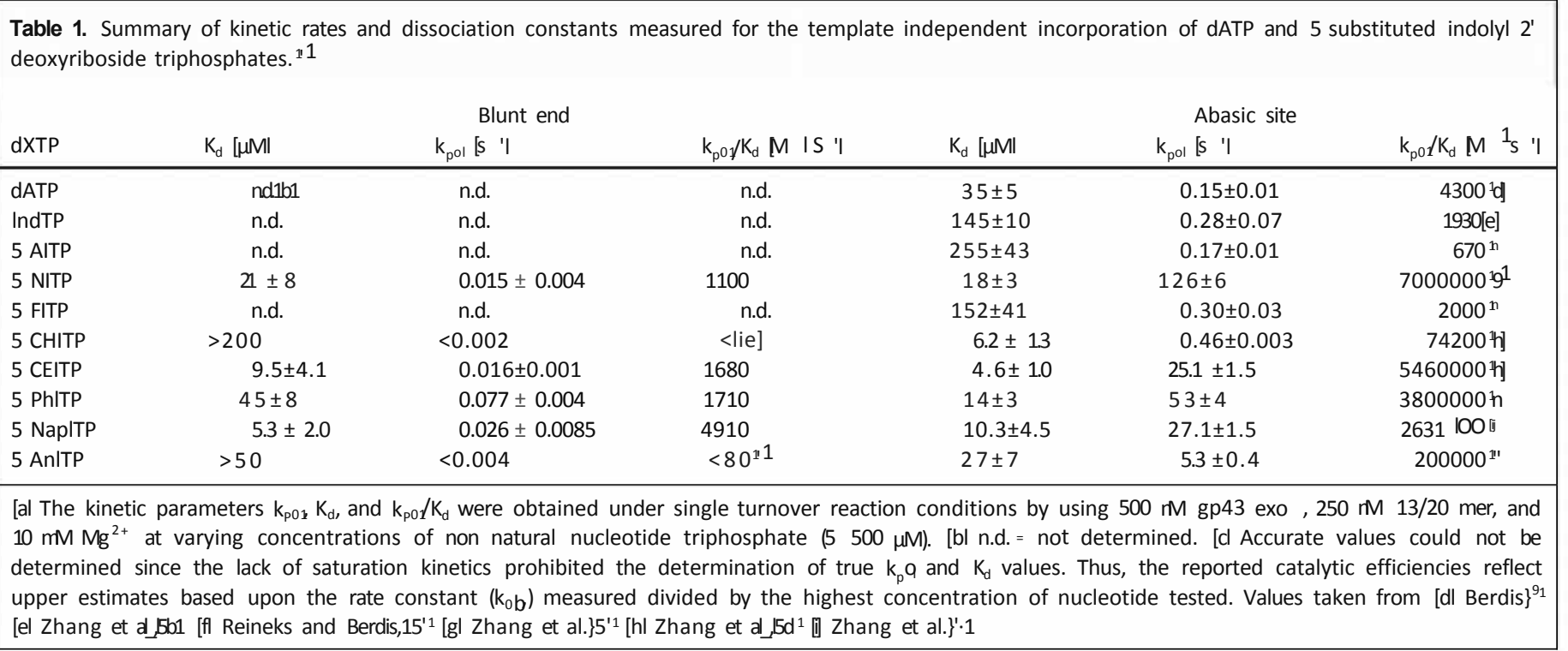

One possible mechanism to explain this trend is that the downstream, single-stranded region of DNA plays an important role in facilitating nucleotide incorporation. This is best exemplified by examining the predicted structures of 5-PhITP paired opposite an abasic site (Figure $3 \mathrm{~A}$ ) or at the blunt-end of DNA (Figure $3 \mathrm{~B}$ ). In each model, the non-natural nucleotide is stacked in an intrahelical position, as expected owing to the large re-electron surface area of the nucleotide. However, the
5-PhITP:abasic site mispair is predicted to be thermodynamically more favorable, since the non-natural nucleobase fills the "void" at the abasic site through dynamic rere stacking interactions with the penultimate A:T base pair as well as with the downstream cytosine residue ( $\mathrm{DNA}_{n}+\mathrm{i}$ position). In contrast, 5PhITP placed at the blunt-end of DNA can only utilize rere stacking interactions with the penultimate A:T pair. Therefore, the lack of stacking interactions with the single-stranded
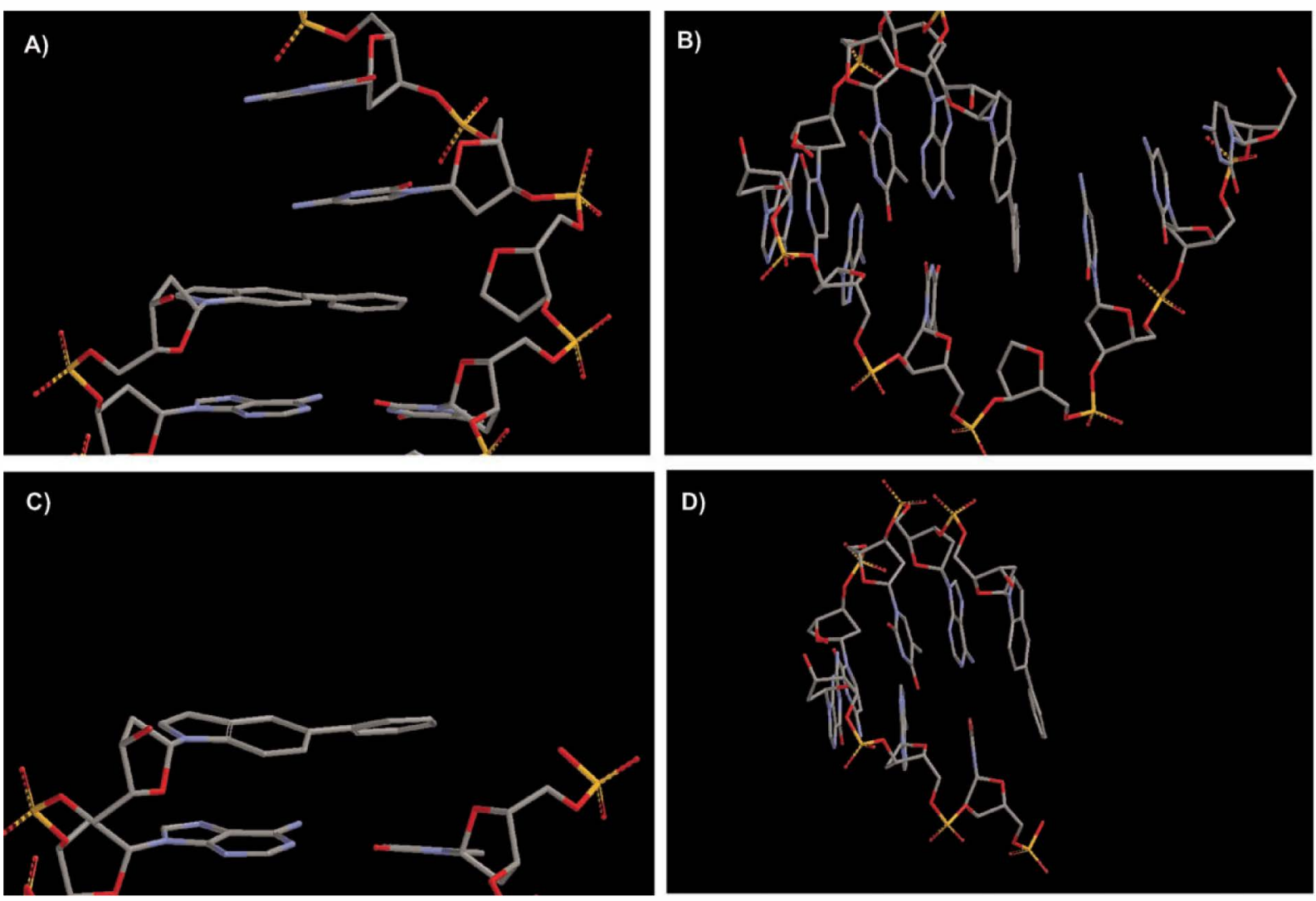

Figure 3. Computer generated models for 5 phenyl indole deoxyribose monophosphate A) and B) paired opposite an abasic site or C) and D) placed at the blunt end of DNA. All models were constructed by using Spartan '04 software according to the manufacturer's protocol. 
region of DNA might cause the slow rate constants for nucleotide incorporation at the blunt-end.

This mechanism was tested by monitoring the incorporation of dATP by using another DNA template, designated 13/20spspmer, that contains two consecutive tetrahydrofuran moieties (Figure 4A). Surprisingly, the incorporation of dATP opposite

A)

$$
\begin{aligned}
& \text { 5'-TCGCAGCCGGTCA } \\
& 3^{\prime}-\text { AGCGTCGGCCAGTXXCCAAA } \quad X=s p
\end{aligned}
$$

B)
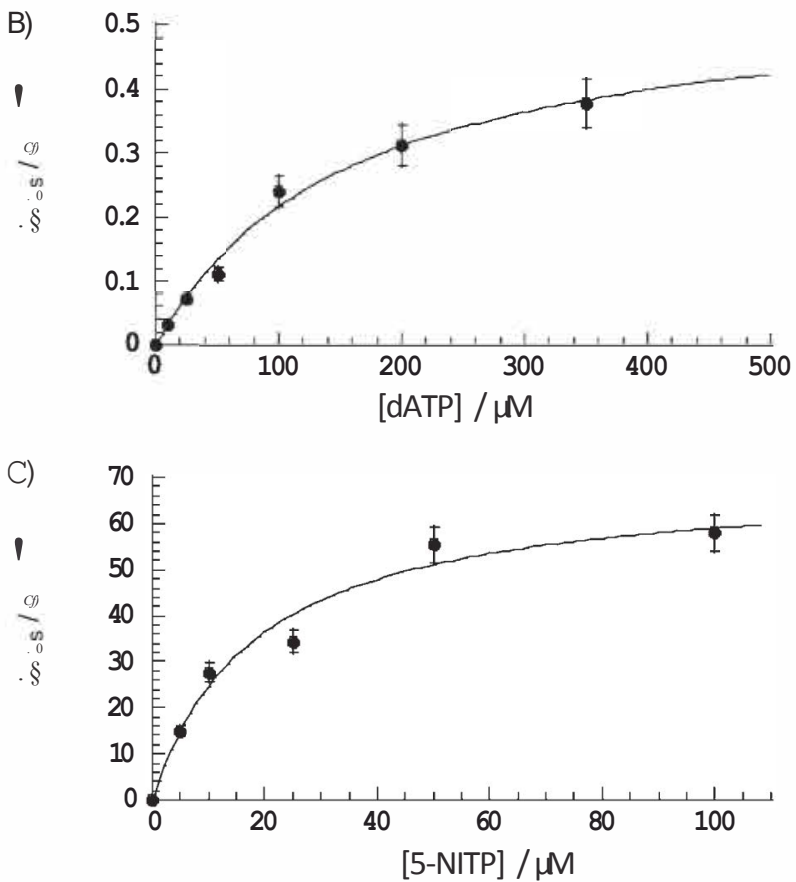

Figure 4. A) Sequence of $13 / 20_{5,5}$, mer DNA substrates used for kinetic anal ysis. "X" in the template strand denotes a tetrahydrofuran moiety designed to functionally mimic an abasic site. B) Michaelis Menten plot for the ob served rate constants in product formation $(\bullet)$ कs a function of dATP con centration. A fit of the data to the Michaelis Menten equation yielded a $K_{d}$ of $157 \pm 28 \mu \mathrm{M}$ and $a \mathrm{k}_{\mathrm{pol}}$ of $0.55 \pm 0.05 \mathrm{~s}^{1 \cdot} \mathrm{C}$ Michaelis Menten plot for the observed rate constants in product formation (e) as a function of 5 NITP concentration. A fit of the data to the Michaelis Menten equation yielded a $\mathrm{K}_{\mathrm{d}}$ of $18 \pm 5 \mu$ Mand $a \mathrm{k}_{\mathrm{pol}}$ of $70 \pm 6 \mathrm{~s}^{1}$.

the damaged DNA is rather facile (data not shown). The $K_{d}$ and kpol values for dATP incorporation were measured under singleturnover reaction conditions, as described earlier. The plot of $\mathrm{k}_{0 \mathrm{~b}}$, versus dATP concentration is hyperbolic (Figure 4B), and a fit of the data to the Michaelis-Menten equation yields a $\mathrm{kpol}$ of $0.55 \pm 0.05 \mathrm{~s}^{-1}$, a $\mathrm{K}_{d}$ of $157 \pm 28 \mu \mathrm{M}$, and a $\mathrm{K}_{\mathrm{p}} / \mathrm{K}_{\mathrm{d}}$ value of $3500 \mathrm{M}_{-}, \mathrm{s}^{-}{ }^{1}$. As predicted, the overall catalytic efficiency for dATP incorporation opposite a "double" abasic site is less than that for incorporation opposite a "single" abasic site. $\left[{ }^{91}\right.$ Howev$\mathrm{er}$, it is interesting that the kpol value of $0.55 \mathrm{~s}^{-1}$ measured opposite the "double" abasic site is - 3.5-fold faster than the value of $0.15 \mathrm{~s}^{-1}$ measured opposite a "single" abasic site. The $K_{d}$ value of $-160 \mu \mathrm{M}$ is, however, nearly five times higher than that measured at a "single" abasic site. ${ }^{[l}$ Thus, it appears that binding affinity for the natural nucleotide decreases as the size of the void increases.

We next measured $K_{d}$ and kpol values for the incorporation of 5-NITP with 13/20spsp-mer as the substrate. The plot of $k_{0 b}$ versus 5-NITP concentration is hyperbolic (Figure $4 \mathrm{C}$ ), and a fit of the data to the Michaelis-Menten equation yields a $\mathrm{kpol}$ of $70 \pm 6 \mathrm{~s}-1, \quad$ a $\mathrm{K}_{d}$ of $18 \pm 5 \mu \mathrm{M}$, and a $\mathrm{Kp}_{\mathrm{a}} / \mathrm{K}_{d}$ value of $3.9 \mathrm{x}$ $10^{6} \mathrm{M}, \mathrm{s}-1$. As was demonstrated above with dATP, the overall catalytic efficiency for 5-NITP incorporation opposite a "double" abasic site is approximately twofold less than opposite a "single" abasic site.[sal In this case, however, the $K_{d}$ value of $18 \mu \mathrm{M}$ is identical to that measured at a "single" abasic site. This result is consistent with our proposed model in which the binding affinity of non-natural nucleotides is independent of the overall size of the void, that is, identical $K_{d}$ values at the blunt end of DNA or at a "single" or "double" abasic site. It should be noted that the kpol value of $70 \mathrm{~s}^{-1}$ measured opposite the "double" abasic site is approximately half the value of $126 \mathrm{~s}-^{1}$ measured opposite a "single" abasic site. Isal Although the effect is minimal, one could argue that the $k_{p 01}$ value decreases as interactions with downstream templating bases are abrogated or at least weakened.

In general, the kinetic data for this new DNA substrate conform well with our model proposing that the lack of stacking interactions with the single-stranded region of DNA could directly influence nucleotide incorporation at the blunt-end. However, an alternative model to explain the lower kpol values during blunt-end extension invokes the contributions of various active-site amino acids during translesion DNA synthesis. Structures of the bacteriophage RB69 DNA polymerase bound at an abasic site $\left.{ }^{{ }_{1}{ }^{0}, 1}\right]_{1}$ or an 8 -oxo-guanine lesion ${ }^{[}{ }_{1} l_{1}$ reveal that two amino acids, G568 and F359, interact significantly with the DNA lesion and downstream template. The structure from Hogg et al. reveals that F359 packs against the base that is immediately $5^{\prime}$ of the abasic site lesion ( $D N A_{n}+i$ position).r,oi It is easy to envision that this aromatic residue provides stacking interactions with the incoming nucleotide that then contribute to the fast kpol values for 5-NITP and 5-PhITP at an abasic site. The kpol values for these nucleotides would be greatly reduced during blunt-end extension, since this aromatic amino acid would not be properly positioned to provide any stabilizing force.

Although not intuitively obvious, G568 might also play a role in positioning the nucleic acid substrate during the polymerization cycle. ${ }_{11}^{1}$ In particular, the structure from Freisinger et al. reveals that G568 lies in close proximity to the templating base $\mathrm{Y}^{11}$ In fact, this amino acid is very mobile, as it reportedly moves - $2 \mathrm{~A}$ depending upon whether a templating base is present or not.r,n The movements of G568 are proposed to reflect the existence of a "strained" versus "relaxed" state of the polymerase:nucleic acid complex that influences the catalytic activity of the enzyme. During blunt-end extension, the inability of G568 to interact effectively with DNA might hinder the rate of the conformation-change step. In either case, it is clear that the interactions of key amino acids with the templating strand play important roles in mediating polymerization during template-dependent or -independent synthesis.

\section{Identifying the rate-limiting step for blunt-end extension}

DNA polymerization occurs according to the multistep pathway illustrated in Figure $5 \mathrm{~A}$ A key feature of this pathway is 
A)

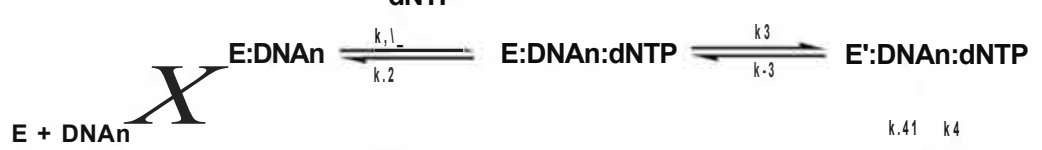

$\mathrm{E}: \mathrm{DNAn+1}=\frac{\mathrm{k} 5}{k . i^{\prime \prime}} \mathrm{E}: \mathrm{DNAn+1:PP}=\frac{\mathrm{ks}}{\mathrm{k.5}} \mathrm{E}: \mathrm{DNAn+1:PP}$

B)
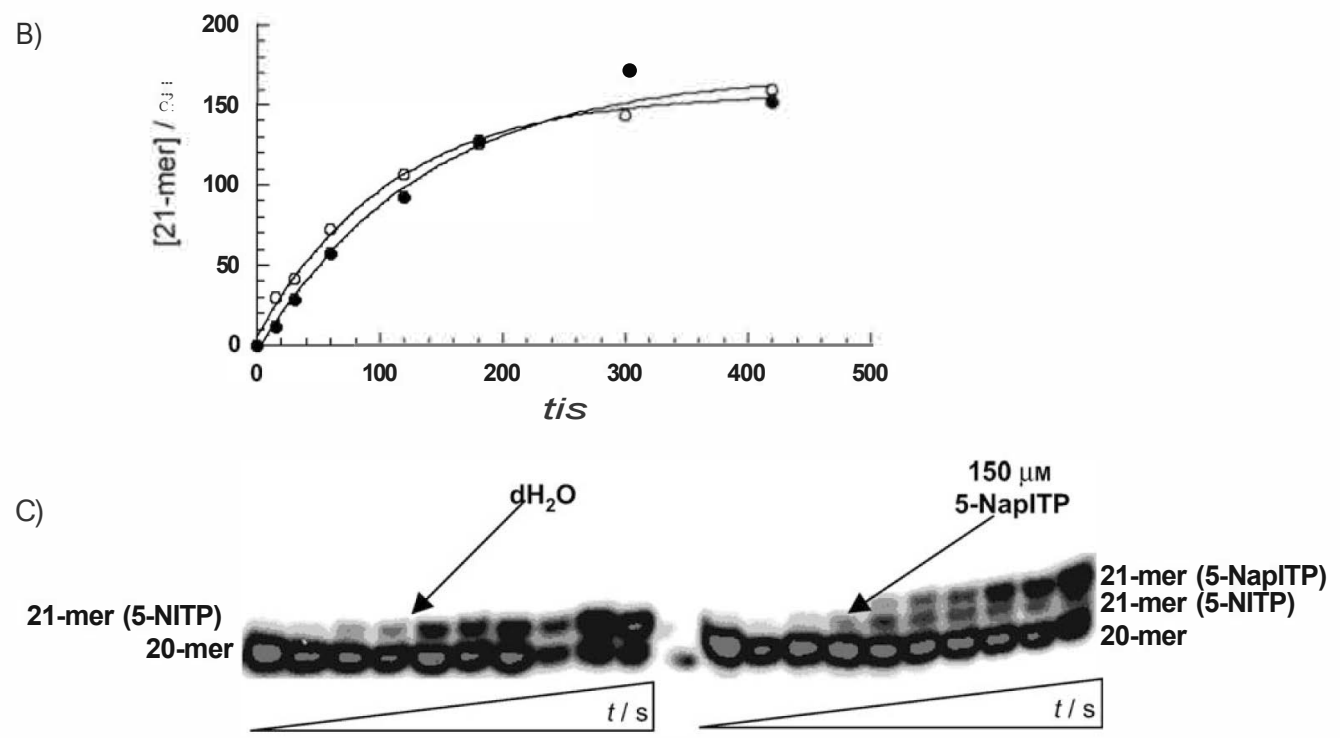

D)

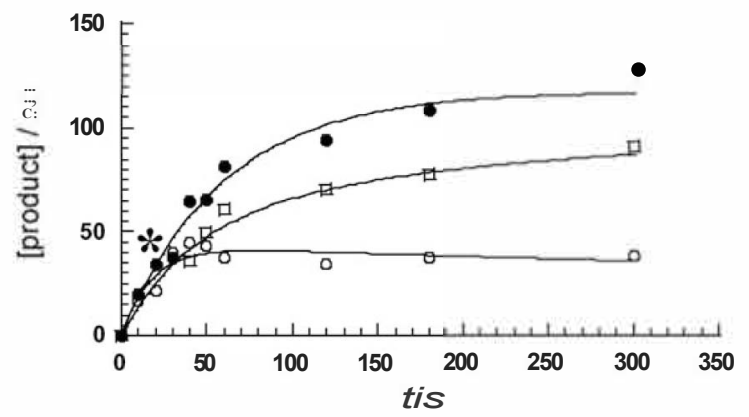

Figure 5. A) Kinetic pathway for DNA polymerization catalyzed by the bacteriophage T4 DNA polymerase. Individ ual steps along the pathway for DNA polymerization are numbered and identified. Abbreviations: $E=T 4$ DNA polymerase, $\mathrm{DNA}_{\mathrm{n}}=\mathrm{DNA}$ substrate, $\mathrm{E}=$ conformational change in DNA polymerase, $\mathrm{PP},=$ inorganic pyrophos phate, and $D N A_{n}+i=$ DNA product (DNA extended by one nucleobase). B) Time courses for the blunt end incorpo ration of 5 NITP with EDTA (o) or HCl (e) as the quenching reagent. Assays were performed as described in the text. Q Gel electrophoresis data for pulse quench (left) versus pulse chase (right) experiments measuring the in corporation of 5 NITP at the blunt end of DNA. Assays were performed as described in the text. D) Quantification of reaction products measured for pulse quench (e) and pulse chase experiments. $\mathrm{h}$ the pulse chase experi ments, 5 NITP incorporation is denoted by o, while 5 NapITP incorporation is denoted by 0 . The asterisk signifies the time point $(30 \mathrm{~s})$ at which the chase nucleotide, $5 \mathrm{NaplTP}$, or $\mathrm{H}_{2} \mathrm{O}$ was added in the pulse chase or pulse quench reaction.

that nucleotide binding occurs in a two-step process in which the first step invokes initial ground-state binding of the nucleotide and the second step represents a conformational change that limits the overall rate of polymerization. Most models of polymerization propose that the conformationalchange step represents the formation of Watson-Crick hydrogen bonds between the incoming nucleotide and the templating nucleobase. ${ }^{\prime} \mathbb{Z}$ The lack of hydrogen-bonding potential at the blunt-end of DNA coupled with the slow rate constants for incorporation of non-natural nucleotides could result from a change in rate-limiting step, so that phosphoryl transfer now becomes rate-limiting. This hypothesis was tested by comparing the kinetics of nucleotide incorporation by using a nondenaturing (EDTA) versus a denaturing $(\mathrm{HCl})$ quenching agent. Any difference in the amount and/or rate constant in product formation as a function of quenching agent can provide information regarding the existence of various enzyme forms, including $E: D N A_{n}, E: D N A_{n}: d N T P$, and $E^{\prime}: D N A_{n}: d N T P$, that accumulate before the phosphoryl transfer step ( $\left.E^{\prime}: \mathrm{DNA}_{n}+1: \mathrm{PP}_{1}\right)$. Time courses for 5-NITP incorporation at a blunt-end under single turnover conditions and a fixed concentration of 5-NITP $(25 \mu \mathrm{M})$ were measured. As shown in Figure $5 \mathrm{~B}$, both the amplitudes and rate constants in product formation are independent of quenching agent; this suggests that the conformational change preceding phosphoryl transfer is the ratelimiting step. Similar mechanistic deductions were made for 5 NITP opposite an abasic site'sal as well as for 5-PhITP incorporation opposite an abasic site $\mathrm{sc}^{\mathrm{rc}}$ or a thymine dimer $/{ }^{61}$ in which nearly identical time courses were obtained regardless of quenching agent.

A series of modified pulsechase experiments was next performed to further confirm that the conformational change is the rate-limiting step for incorporation at the blunt-end of DNA. In these experiments, the E:DNA complex was first "pulsed" with a subsaturating concentration of 5-NITP $(25 \mu \mathrm{M})$ to form the E:DNA $: 5-N I T P$ complex. This species was either quenched with EDTA or "chased" with a saturating concentration $(150 \mu \mathrm{M})$ of 5-NapITP. 5-NITP and 5-NapITP were used so that the incorporation of either non-natural nucleotide could be resolved by denaturing gel electrophoresis (Figure $5 \mathrm{C}$ ). In the pulse-chase experiment, 5-NITP is incorporated at the blunt-end of the DNA as represented by the conversion of 20mer to 21-mer. The pulse-chase reaction illustrated on the right in Figure $5 \mathrm{C}$ reveals the presence of two distinct product 
bands, which reflect the incorporation of 5-NITP (lower band) and 5-NapITP (higher band). Under these reaction conditions, 5-NapITP can only be incorporated if any bound 5-NITP dissociates from the E:DNA:5-NITP complex prior to the phosphoryl transfer step. The data provided in Figure 5D clearly demonstrates that the amount of 5-NITP incorporation remains invariant after the addition of 5-NapITP. These data collectively argue that the conformational-change step is rate limiting for incorporation. The kinetic mechanism for DNA polymerization illustrated in Figure 5A is used to further support this argument. For example, 5-NaplTP is expected to be incorporated as the "chase" if the off-rate for 5-NITP (k_2) is faster than the rate constant for the conformational-change step $\left(k_{3}\right)$. Thus, these data indicate rapid equilibrium binding of 5-NITP to the E:DNA complex since the conformational change is the rate-limiting step. This is validated mathematically as the calculated off-rate for 5-NITP ranges between 0.25 and $2.5 \mathrm{~s}-1$ and is at least ten times faster than the $k_{\text {pol }}$ value of $0.0,5 \mathrm{~s}-1,131$

This conclusion has several important ramifications for the molecular mechanism of polymerization. First, it is clear that gp43 does not require hydrogen-bonding interactions to trigger the conformational change, since this step limits nucleotide incorporation during template-independent DNA synthesis. This implies that some other biophysical feature(s) must be used to provide the energy needed to facilitate this step. The data presented in Table 1 argue that the n-electron surface area of the incoming nucleotide is an important feature used to facilitate this kinetic step. However, it is clear that the environment of the primer template also plays an important role, since the magnitude of the $\mathrm{k}_{\mathrm{pol}}$ values is dependent upon the nature of the nontemplating lesion. As before, the rate constants for incorporation at the blunt end of DNA are much slower than those measured at an abasic site, despite the fact that both cases are measuring template-independent DNA synthesis.

\section{Template-independent DNA synthesis by terminal deoxy- nucleotidyl transferase}

Mammalian terminal deoxyribonucleotidyl transferase (TdTase) catalyzes the non-template-directed polymerization of dNTPs using only single-stranded DNA as the substrate.[141 This enzyme activity contrasts with all other DNA polymerases, which have an absolute requirement for a DNA template-or at least duplex DNA containing a free 3 '-OH (vide supra). This unique activity provides another distinct opportunity to evaluate the contributions of biophysical forces other than hydrogen bonding and steric constraints to the efficiency of DNA polymerization.

To evaluate these features, we measured the ability of TdTase to incorporate natural and non-natural nucleotides using single-stranded DNA as the substrate. Representative data provided in Figure 6A reveal that TdTase incorporates all natural nucleotides in a template-independent fashion. The ladder in polymerization products provides evidence that TdTase incorporates and extends each natural nucleotide. In general, longer products are generated with dGTP and dCTP than with equivalent concentrations of dATP or dTIP. Quantification of the gel electrophoresis data reveals that the total amount of primer elongation is greatest with dCTP and dGTP. Furthermore, the kinetics of elongation are optimal for dCTP and dGTP rather than for dATP or dTIP. Collectively, these data suggest that dGTP and dCTP are preferentially utilized and are consistent with the previously published reports from the Modak $^{[15}$ and Coleman ${ }^{[16]}$ laboratories.

We next measured the ability of TdTase to utilize various non-natural nucleotides. Representative gel electrophoresis data (Figure 6B) reveal that TdTase effectively utilize nearly every member of our library of non-natural nucleotides. The lone exception is 5-AITP, which is poorly incorporated even at concentrations greater than $500 \mu \mathrm{M}$ (data not shown). Nonetheless, the relatively facile incorporation of these diverse nucleotides indicates that TdTase does not rely solely upon hydrogen-bonding interactions for catalyzing template-independent polymerization. It should be noted that certain non-natural nucleotides are used more efficiently than others. For example, IndTP and 5-FITP yield smaller extension products (ranging from $\mathrm{DNA}_{n}+1$ to $\mathrm{DNA}_{n}+{ }_{s}$ compared to 5-NITP, which shows a larger range of elongation products (up to $\mathrm{DNA}_{n}+11$ - The ability of TdTase to elongate these non-natural nucleotides is surprising, since these analogues are potent chain terminators of DNA synthesis when incorporated into duplex DNA by gp43.[s] The kinetics of extension do not appear to be influenced by nelectron surface area, since other analogues, such as 5-PhITP, 5-CEITP, and 5-NapITP, are incorporated but not efficiently elongated. In fact, smaller analogues, such as 5-FITP and 5-NITP, are more efficiently elongated whereas their bulkier counterparts are refractory to elongation. The inability to elongate large, bulky, non-natural nucleotides likely results from steric constraints imposed by structural features present within this unique polymerase. Structural studies of the murine TdTase reveal the presence of a 16-amino-acid loop that could function as a steric gate to provide specificity for single-stranded versus duplex DNA. [ ${ }^{1}$ Regardless, the data presented here indicate that template-independent synthesis catalyzed by TdTase can occur in the absence or presence of hydrogenbonding interactions. It will prove interesting to further characterize the kinetic behavior of TdTase to evaluate which biophysical features are utilized by this enigmatic polymerase.

\section{Conclusions}

This report describes the mechanism by which the various DNA polymerases perform template-independent DNA polymerization. This type of activity has been previously reported for members of the X-family of DNA polymerases. ${ }^{[181}$ However, this represents the first report comparing blunt-end extension versus replication opposite a nontemplating DNA lesion by using a high-fidelity DNA polymerase. In this case, the T4 DNA polymerase can only perform blunt-end extension using nonnatural nucleotides that have strong base-stacking capabilities. The $K_{d}$ values for these are essentially identical during bluntend extension versus incorporation at an abasic site and suggest that the T4 DNA polymerase uses n-electron density as 
A)
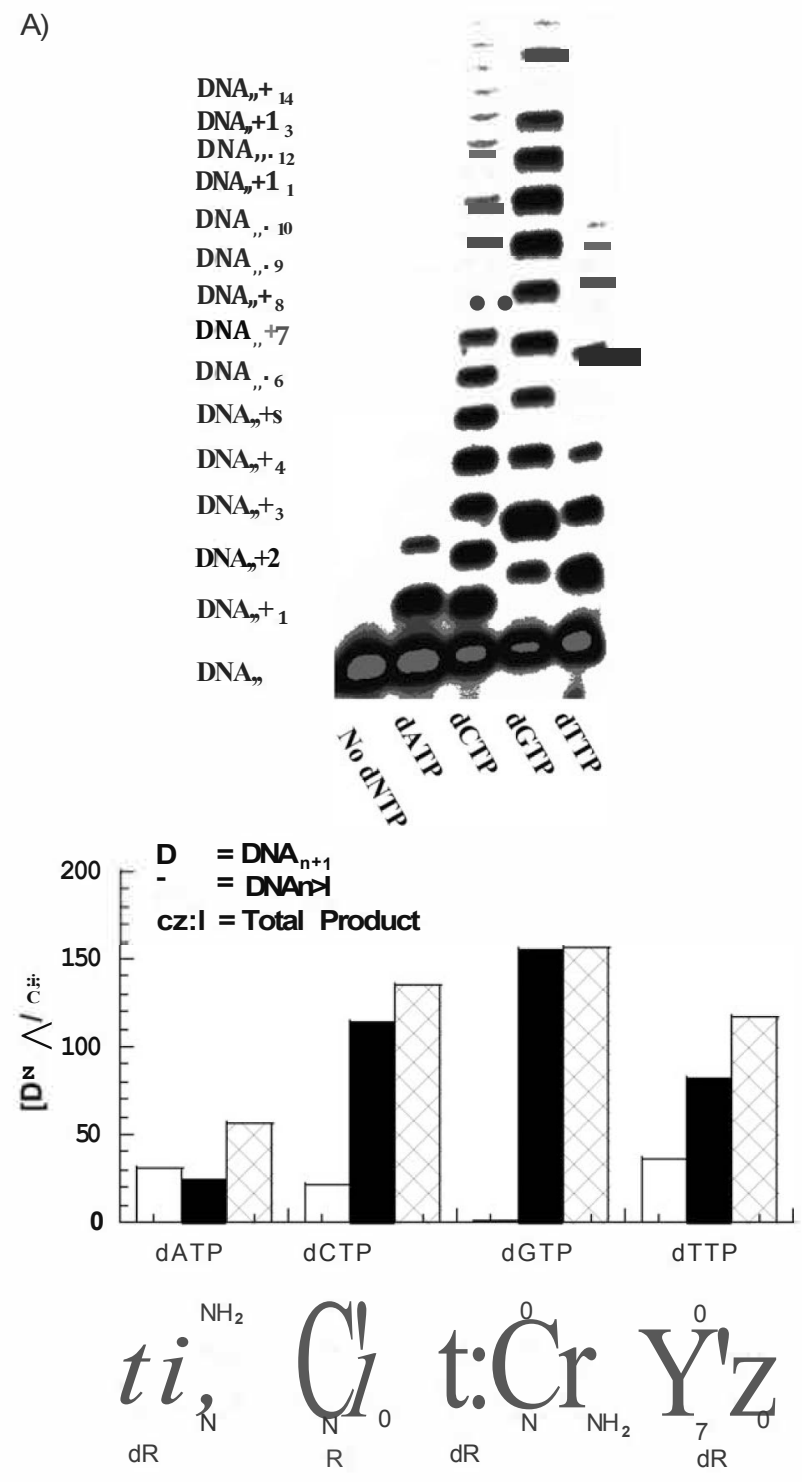

B)
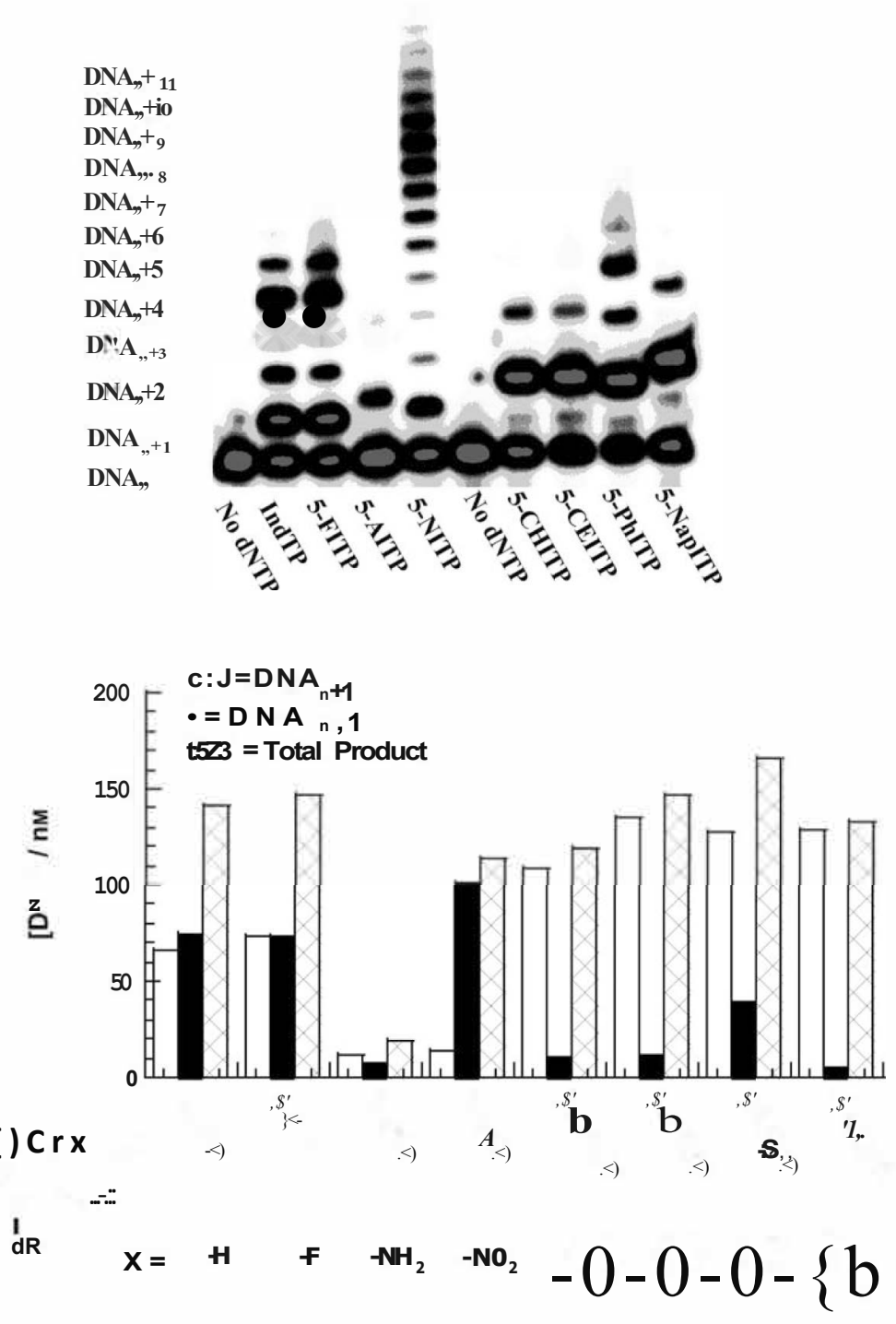

Figure 6. Gel electrophoresis data comparing the ability of terminal deoxynucleotidyl transferase (TdTase) to utilize A) natural or B) non natural nucleoside tri phosphates. Assays were performed as described in the text.

the molecular force to stabilize ground-state binding interactions in the absence of templating information. However, the large differences in the $\mathrm{k}_{\mathrm{pol}}$ values measured during incorporation opposite an abasic site versus blunt-end DNA suggest that the single-stranded region of the DNA template plays an important role by facilitating the conformational-change step preceding phosphoryl transfer. At the molecular level, this might include stacking interactions with downstream bases, interactions with key amino acid residues, or a combination of the two. Additional support for this mechanism is provided by the recent NMR structures of pyrene triphosphate, a non-natural nucleotide similar to 5-PhITP, when it is paired opposite an abasic site ${ }^{91}$

In addition, we have demonstrated that TdTase, a templateindependent DNA polymerase, can incorporate many of these non-natural nucleotides. Although the majority of these analogues are incorporated efficiently, there are significant differ- ences in the extension of these non-natural nucleotides that depend primarily on their overall size. Surprisingly, 5-NITP is the most efficiently extended analogue; a contrast with its chain-termination activity during template-dependent and template-independent DNA synthesis catalyzed by the T4 DNA polymerase. The ability of TdTase to utilize these analogues has interesting ramifications for the development of chemotherapeutic agents. For example, TdTase plays a key role in $\mathrm{V}(\mathrm{D}) \mathrm{J}$ recombination during lymphocyte and repertoire development.120 $0^{1}$ However, TdTase activity is also reported to be elevated in acute lymphocytic leukemia (ALL) and during the crisis stage of chronic myeloid leukemia (CML). $\left[21^{1}\right.$ Both findings coincide with poor responses to chemotherapy, and generally lead to reduced survival expectancy. $122^{1}$ it will be interesting to evaluate if these non-natural nucleotide can selectively inhibit TdTase activity and provide a novel class of anticancer agent that targets these forms of leukemia. 


\section{Experimental Section}

Materials: [y-32P]ATP was purchased from MP. Biomedical (Irvine, CA). Unlabeled dNTPs (ultrapure) were obtained from Pharmacia. MgAcetate and Trizma base were from Sigma. Urea, acrylamide, and bis-acrylamide were from Aldrich. Oligonucleotides, including those containing 5-nitroindole, were synthesized by Operon Technologies (Alameda, CA). Single-stranded and duplex DNA were purified and quantified as described $Y^{3 l}$ All other materials were obtained from commercial sources and were of the highest available quality. The exonuclease-deficient mutant of gp43 (Asp219Ala) was purified and quantified as previously described $Y^{4 \mid}$ The non-natural nucleotides used in this study were synthesized and purified as previously described_15!

Enzyme assays: The assay buffer used in all kinetic studies consisted of Tris-OAc (25 mM; pH 7.5), KOAc (150 mM), and 2-mercaptoethanol $(10 \mathrm{mM})$. All assays were performed at $25^{\circ} \mathrm{C}$. Polymerization reactions were monitored by analysis of the products on $20 \%$ sequencing gels, as previously described $\mathrm{Y}^{31}$ Gel images were obtained with a Packard Phosphorlmager by using the OptiQuant software supplied by the manufacturer. Product formation was quantified by measuring the ratio of $32 \mathrm{P}$-labeled extended and nonextended primer. The ratios of product formation were corrected for substrate in the absence of polymerase (zero point). Corrected ratios were then multiplied by the concentration of primer/template used in each assay to yield total product. All concentrations are listed $a$ final solution concentrations.

Nucleotide incorporation assays: Experiments were performed under single-turnover reaction conditions in which gp43 exo $(500 \mathrm{nM})$ was incubated with DNA $(250 \mathrm{nM})$ in assay buffer containing EDTA $(100 \mu \mathrm{M})$ and mixed with variable concentrations of nucleotide analogue $(5500 \mu \mathrm{M})$ and $\mathrm{Mg}(\mathrm{OAch}(10 \mathrm{mM})$. The reactions were quenched with EDTA $(200 \mathrm{mM})$ at various times $(5600 \mathrm{~s})$ and analyzed as described above. Data obtained for single turnover DNA polymerization assays were fitted to Equation (1).

y $\quad A\left(l \quad k^{\prime}\right)+C$

Here $A$ is the burst amplitude, $k$ is the observed rate constant $\left(k_{b,}\right)$ in initial product formation, $\mathrm{t}$ is time, and $\mathrm{C}$ is a defined constant. Data for the dependency of $k_{\mathbf{o}}$, as a function of dNTP concentration were fitted to the Michaelis Menten equation [Eq. (2)] to provide values corresponding to $\mathrm{k}_{\mathrm{pol}}$ and $\mathrm{K}_{\mathrm{d}}$.

$k_{\text {obs }} \frac{\mathrm{k}_{\mathrm{p} 01}[\mathrm{dXTP}]}{\mathrm{K}_{\mathrm{d}}+[\mathrm{dXTP}]}$

Here $k_{\mathbf{b}}$, is the observed rate constant of the reaction, $k_{p o l}$ is the maximal polymerization rate constant, $K_{d}$ is the dissociation constant for DXTP, and dXTP is the concentration of non-natural nucleotide substrate.

Acid versus EDTA as the quenching agent: Duplex DNA (250 nM) was incubated with $K_{t}$ concentrations of non-natural nucleotide and $\mathrm{Mg}(\mathrm{OAch}(10 \mathrm{mM})$. The reaction was then initiated with gp43 exo $(1 \mu \mathrm{M})$ and quenched with either EDTA $(500 \mathrm{mM})$ or HCl $(1 \mathrm{M})$ at time intervals ranging from 5 to $600 \mathrm{~s}$ as described above. After the reaction had been quenched with $\mathrm{HC}(1 \mathrm{M})$, phenol/chloroform/iso-amyl alcohol $(100 \mu \mathrm{L})$ was added to extract the DNA polymerase, and the $\mathrm{pH}$ of the aqueous phase was neutralized by addition of $1 \mathrm{M}$ Tris $/ 3 \mathrm{M} \mathrm{NaOH}(-30 \mu \mathrm{L})$. Product formation was analyzed and quantified as described above.
Modified pulse-chase experiments: gp43 exo $(1 \mu \mathrm{M})$ was preincubated with DNA $(250 \mathrm{nM})$ and $\mathrm{Mg}(\mathrm{OAc})_{2}(10 \mathrm{mM})$ followed by the addition of 5-NITP $(25 \mu \mathrm{M})$ as the "pulse". $\mathbf{n}$ the "chase" experiment, 5-NapITP $\left(150 \mu \mathrm{M} ; 10 \times \mathrm{K}_{\mathrm{d}}\right.$ concentration) was added after $30 \mathrm{~s}$ (approximately one half-life for the blunt-end incorporation of 5 -NITP). Aliquots of the reaction mixtures were quenched with EDTA (200 mM) at times points prior to $\left(\begin{array}{ll}0 & 10 \mathrm{~s}\end{array}\right)$ and after the addition of the 5-NapITP "chase" ( M $10300 \mathrm{~s}$ ). Product formation was quantified as described above. Due to the difference in size between 5-NITP and 5-NapITP, both reaction products could be adequately separated by denaturing gel electrophoresis. Data were fitted to the equation for a single-exponential process [Eq. (1)] or to Equation (3), which describes a burst followed by a slow, steadystate phase.

y $A\left(l \quad k^{\prime}\right)+B t+C$

Here $A$ is the burst amplitude, $k$ is the observed rate constant $\left(k_{\mathbf{b},}\right)$ in initial product formation, $t$ is time, $B$ is the steady-state rate, and $C$ is a defined constant.

\section{Acknowledgements}

This research was supported by funding from the National Institutes of Health to A.J.B. (CA 778408).

Keywords: base-stacking - catalysis - DNA polymerization • nucleotides $\cdot$ pi interactions

[1] a) M F. Goodman, K D. Fygenson, Genetics 1998, 748, 1475 1482; b) T. A. Kunkel, K Bebenek, Annu. Rev. Biochem. 2000, 69, 497 529; c) M T. Washington, S. A. Helquist, E.T. Kool, L Prakash, S. Prakash, Mo/. Cell. Biol. 2003, 23, 5107 5112; d) J.M. Krahn, W. A. Beard, S. H. Wilson, Structure 2004, 72, 18231832.

[2] a) P. Zhang, W. T. Johnson, D. Klewer, N. Paul, G. Hoops, V. J. Davisson, D. E Bergstrom, Nucleic Acids Res. 1998, 26, 2208 2215; b) N. Paul, V. C. Nashine, G. Hoops, P. Zhang, J. Zhou, D. E Bergstrom, V. J. Davisson, Chem. Biol. 2003, 70, 815 825; c) 0. Adelfinskaya, V. C. Nashine, D. E Bergstrom, V. J. Davisson, $\perp$ Am. Chem. Soc. 2005, 727, 1600016001.

[3] a) A. K Ogawa, Y. Wu, M Berger, P. G. Schultz, F. E Romesberg, $\perp \mathrm{Am}$. Chem. Soc. 2000, 722, 88038804 ; b) Y. Wu, A. K Ogawa, M Berger, D. L. McMinn, P. G. Schultz, F. E Romesberg, I Am. Chem. Soc. 2000, 722, 7621 7632; c) E L Tae, Y. Wu, G. Xia, P. G. Schultz, F. E Romesberg, $\perp \mathrm{Am}$. Chem. Soc. 2001, 723, 74397440.

[4] a) S. Moran, R X. Ren, E.T. Kool, Proc. Natl. Acad. Sci. USA 1997, 94, 10506 10511; b) E.T. Kool, Annu. Rev. Biochem. 2002, 77, 191 219; c) J.C. Morales, E.T. Kool, Nat. Struct. Biol. 1998, 5, 950 954; d) T. W. Kim, L G. Brieba, T. Ellenberger, E.T. Kool, J. Biol. Chem. 2006, 287, 2289 2295; e) S. Mizukami, T. W. Kim, S. A. Helquist, E.T. Kool, Biochem istry 2006, 45, 2772 2778; f) H. 0. Sintim, E.T. Kool, Angew. Chem. 2006, 778, 2008 2013; Angew. Chem. Int. Ed. 2006, 45, 19741979.

[SJ a) E Z. Reineks, A. J. Berdis, Biochemistry 2004, 43, 393 404; b) X. Zhang, I Lee, A. J. Berdis, Org. Biomol. Chem. 2004, 2, 1703 1711; c) X. Zhang, I Lee, A. J. Berdis, Biochemistry 2005, 44, 13101 13110; d) X. Zhang, X. Zhou, I Lee, A. J. Berdis, J. Am. Chem. Soc. 2006, 728, 143 149; e) X. Zhang, A. Donnelly, I Lee, A. J. Berdis, Biochemistry 2006, 45, 1329313303.

[6] B. Devadoss, I Lee, A. J. Berdis, Biochemistry 2007, 46, 44864498.

[7] Since single turnover conditions were used in these studies, the $k_{p o l}$ value reflects the rate constant for the first round of DNA polymeri zation and should not be confused with the more common term, k"" which defines the rate constant measured during multiple rounds of polymerase turnover.

[8] T. L Capson, J. A. Peliska, B. F. Kaboord, M W. Frey, C. Lively, M Dahl berg, S. J. Benkovic, Biochemistry 1992, 37, 1098410994. 
[9] M W. Frey, N G. Nossal, T. L. Capson, S. J. Benkovic, Proc. Natl. Acad. Sci. USA 1993, 90, 25792583.

11 O As reported in Table 1 , the $\mathrm{K}_{0}$ of $45 \mu \mathrm{M}$ for $5 \mathrm{PhlTP}$ incorporation at the blunt end of DNA is three times higher than that measured opposite the abasic site. The reason for the difference in binding affinity is un known.

[IIJ A. J. Berdis, Biochemistry 2001, 40, 71807191.

[12 M Hogg, S. S. Wallace, S. Doublie, EMBO J. 2004, 23, 14831493.

[13] E Freisinger, A. P. Grollman, H. Miller, C. Kisker, EMBO J. 2004, 23, 1494 1505.

[14J K A Johnson, Annu. Rev. Biochem. 1993, 62, 685713.

IISJ The $k_{\text {off }}$ values ( $k_{2}$ ) of $0.252 .5 \mathrm{~s} 1_{\text {for }} 5$ NITP were calculated from the equation $K_{d}=k_{0} f ! k_{00}$ by using the measured $K_{d}$ value of $25 \mu \mathrm{M}$ with the assumption that the $k_{00}$ values ranges from $10^{5} 10^{6} \mathrm{M}{ }^{1} \mathrm{~s}^{1}$

[16] F. J. Bollum, Adv. Enzymol. Re/at. Areas Mo/. Biol. 1978, 47, 347374.

[17J a) M Basu, M V. Hegde, M J. Modak, Biochem. Biophys. Res. Commun. 1983, 77, 1105 1112; b) V. Pandey, M J. Modak, Prep. Biochem. 1987, 77, 359 377; c) V. N Pandey, M J. Modak, J. Biol. Chem. 1989, 264, 867 871.

[18J a) S. A. Fuller, A. Philips, M S. Coleman, Biochem. J. 1985, 231, 105 113; b) B. Yang, K N. Gathy, M S. Coleman, J. Biol. Chem. 1994, 269, 11859 11868 .
[19] M Delarue, J. B Boule, J. Lescar, N Expert Bezan on, N Jourdan, N Sukumar, F. Rougeon, C. Papanicolaou, EMBO J. 2002, 27,427 439.

[20] a) E C. Friedberg, P. L Fischhaber, C. Kisker, Cell 2001, 107, 9 12; b) S. A. Nick McElhinny, D. A. Ramsden, Immunol. Rev. 2004, 200, 156 164; c) M Garcia Diaz, K Bebenek, G. Gao, L C. Pedersen, R E London, T. A. Kunkel, DNA Repair 2005, 4, 13581367.

[21 S. Smirnov, T. J. Matray, E.T. Kool, C. de Los Santos, Nucleic Acids Res. 2002, 30, 55615569.

[22 C. L. Benedict, S. Gilfillan, T. H. Thai, J. F. Kearney, Immunol. Rev. 2000, 775, 150157.

[23] a) P. Patmasiriwat, T. Anukarahanonta, S. Chinprasertsuk, Ann. Clin. Lab. Sci. 1993, 23, 281 289; b) E Paietta, J. Racevskis, J.M. Bennett, P.H. Wiernik, Br. J. Haemato/. 1993, 84,416 422; c) S. C. Wolf, P. G. Steinherz, N R Landau, A E Silverstone, Am. J. Hemato/. 1987, 25,259 269.

[24J J. M Bhatavdekar, S. N Trivedi. H. H. Vora, S. N Shukla, Int. J. Biol. Mark ers $1993,8,7780$ 